\title{
The BENEFIT trial: testing the hypothesis that trypanocidal therapy is beneficial for patients with chronic Chagas heart disease
}

\author{
J Antonio Marin-Neto $/^{1+}$, Anis Rassi Jr², Alvaro Avezum Jr ${ }^{3}$, Antonio C Mattos ${ }^{3}$, Anis Rassi ${ }^{2}$ \\ 'Divisão de Cardiologia, Departamento de Medicina Interna, Faculdade de Medicina de Ribeirão Preto, Universidade de São Paulo, \\ Ribeirão Preto, SP, Brasil ' Hospital Anis Rassi, Goiânia, GO, Brasil ${ }^{3}$ Instituto Dante Pazzanese de Cardiologia, São Paulo, SP, Brasil
}

\begin{abstract}
Among the pathophysiological derangements operating in the chronic phase of Chagas disease, parasite persistence is likely to constitute the main mechanism of myocardial injury in patients with chronic chagasic cardiomyopathy. The presence of Trypanosoma cruzi in the heart causes a low-grade, but relentless, inflammatory process and induces myocardial autoimmune injury. These facts suggest that trypanocidal therapy may positively impact the clinical course of patients with chronic Chagas heart disease. However, the experimental and clinical evidence currently available is insufficient to support the routine use of etiologic treatment in these patients. The BENEFIT project - Benznidazole Evaluation for Interrupting Trypanosomiasis - is an international, multicenter, double-blind, placebo-controlled trial of trypanocidal treatment with benznidazole in patients with chronic Chagas heart disease. This project is actually comprised of two studies. The pilot study investigates whether etiologic treatment significantly reduces parasite burden, as assessed by polymerase chain reaction-based techniques and also determines the safety and tolerability profile of the trypanocidal drug in this type of chagasic population. The full-scale study determines whether antitrypanosomal therapy with benznidazole reduces mortality and other major cardiovascular clinical outcomes in patients with chronic Chagas heart disease.
\end{abstract}

Key words: Chagas disease - Chagas cardiomyopathy - antitrypanosomal therapy - benznidazole - clinical trials

Unfortunately, one century after its discovery (Chagas 1909), Chagas disease still affects millions of poor people in several Latin American countries and is a leading cause of sudden death, severe cardiac arrhythmias and intractable heart failure (Dias et al. 2008). In addition, the disease cannot be prevented by vaccination or, in many situations, be reliably cured by antiparasitic drugs. Various epidemiological features of the disease require appropriate evaluation, including recent outbreaks of acute infection in the Amazon Region (Coura 2007). Long after the initial phase, the only period when the etiologic agent is readily found, clinical complications of the chronic phase appear in at least $30-40 \%$ of infected people. So far, there is no way to predict which patients will develop these chronic complications (Dias 1989), nor do we understand the mechanisms leading to many of them, including myocardial damage (Marin-Neto et al. 2007). One possibility is that autonomic nervous system disturbances (Köberle 1968) cause ischemic myocardial insult or trigger ventricular arrhythmias, both of which can cause sudden death (Amorim \& Marin-Neto 1995). Also, regional perfusion disturbances and abnormal control of the coronary microcirculation are likely to play at least an ancillary role in myocardial fibre destruction, which leads to fibrotic replacement (Rossi 1990, MarinNeto et al. 1992). Myocardial autoimmune inflamma-

+ Corresponding author: marin_neto@yahoo.com

Received 26 May 2009

Accepted 8 June 2009 tion also occurs in both experimental and clinical cases of chronic Chagas cardiomyopathy (Cunha-Neto et al. 2006, Kierszenbaum 2007). Studies have shown that the low-grade, incessant myocarditis seen in the chronic phase of Chagas disease is related to parasite persistence (Bellotti et al. 1996, Tarleton 2003) and much of the immune-mediated myocardial inflammation may also be parasite-driven. Therefore, eliminating the parasite, or at least reducing its burden, may favourably impact the natural history of chronic Chagas disease.

\section{Trypanocidal drugs used in Chagas disease}

Chagas disease continues to be a rather neglected morbid entity, as illustrated by the fact that for more than three decades, only two drugs, nifurtimox and benznidazole, have been available to effectively treat Trypanosoma cruzi. Nifurtimox, a nitrofuran derivative (Lampit, Bayer 2503, Leverkusen, Germany), has been extensively used for over three decades, but is currently not available in several countries, including Brazil. Benznidazole (LAFEPE, Recife, Brazil), a nitroimidazole derivative, has also been widely used as an effective agent for antiparasitic therapy in cases of acute or sub-acute Chagas disease, including transfusion and laboratory-acquired disease and reactivation of infection in transplanted and other immunosuppressed patients.

\section{Trypanocidal therapy in acute Chagas disease}

In the acute stages of Chagas infection, nifurtimox reduces the severity and duration of the illness and may possibly reduce mortality. Parasitological cure (permanent negative xenodiagnosis and serology) occurs in about $70 \%$ of treated patients. The main drawback of nifurtimox therapy is the high incidence of side ef- 
fects, which are reported in up to $40 \%$ of patients. Side effects with nifurtimox usually include gastrointestinal complaints, such as nausea, vomiting, abdominal pain, weight loss and severe anorexia. Neurologic adverse effects may also occur and include restlessness, paresthesias, twitching, insomnia and seizures. These symptoms generally resolve when the dosage is reduced or therapy is discontinued. Therapy with benznidazole has a similar efficacy profile to nifurtimox, with the advantage of a lower side-effect rate. Treatment with benznidazole in cases of acute Chagas disease produces long-term parasitologic cure in approximately $70 \%$ of patients. In one study, 43 patients treated in the acute phase of Chagas disease with benznidazole or nifurtimox were followed for several years (Rassi et al. 2000). The incidence of chronic clinical manifestations of the disease was higher in patients whose serology was positive after treatment (36\%) compared to those patients with long-term negative serology $(7 \%)$.

No randomised studies have examined the effect of benznidazole on significant clinical long term outcomes of patients treated during the acute phase of Chagas disease. Despite the lack of such evidence, it is generally accepted that antiparasitic therapy should be administered to all patients diagnosed with acute Chagas disease, regardless of the mechanism of infection. Furthermore, this treatment should also be initiated in all cases of disease reactivation in chronic patients (Rassi et al. 2000, Bern et al. 2007).

\section{Trypanocidal therapy in chronic Chagas disease}

The role of antiparasitic treatment in the chronic phase of Chagas disease is more controversial, as evidence from studies, either using experimental models of the disease or following chronic Chagas patients, is scarce. Data suggest that from a serological and parasitological standpoint, the cure rates of treatment are highly variable in the chronic phase of the disease and depend on the timing of treatment, with higher rates seen in those treated earlier in the disease. These data also indicate that following the trypanocidal therapy, there is a gradual decrease in the serological titres of antitrypanosoma antibodies, and that treatment failure may sometimes manifest, even decades after persistently negative xenodiagnostic tests.

\section{Evidence of benefit with trypanocidal therapy in ex- perimental models of chronic Chagas disease}

There is some evidence that etiologic treatment in animals infected with the T. cruzi may attenuate the course of myocarditis in the chronic phase of Chagas disease. One study showed that in mice chronically infected with various strains of $T$. cruzi, treatment with benznidazole or nifurtimox had significantly less myocardial damage, as assessed by histopathology, than untreated infected mice (Andrade et al. 1991).

A more recent study demonstrated several beneficial effects of benznidazole treatment in mice chronically infected with the Colombian T. cruzi. Compared to untreated mice, the hearts of treated mice had less parasitism and myocarditis. Treatment also led to less cardiac conduction disturbances and ventricular extrasystoles in the electrocardiogram (ECG) of treated animals, as well as lower serum levels of antibodies against $\mathrm{T}$. cruzi antigens (epimastigote extract, $\mathrm{P}_{2} \beta$, and trans-sialidase). Decreased antibodies against peptides of the second extracellular loops of $\beta_{1}$-adrenergic and $\mathrm{M}_{2}$-muscarinic cardiac receptors in benznidazole-treated mice were also seen. The investigators concluded that etiologic treatment in the chronic phase of infection prevented the development of severe chronic cardiomyopathy, despite the lack of complete parasite eradication (Garcia et al. 2005).

\section{Evidence of benefit with trypanocidal therapy in hu- man chronic Chagas disease}

While there have been both observational and randomised controlled studies, no conclusions can be definitively drawn from these studies. Overall, clinically significant endpoints were included in several nonrandomised studies, but the conclusions were hindered by their observational design. Conversely, the randomised studies failed to include hard endpoints, limiting their clinical relevance.

\section{Evidence from non-randomised clinical trials}

Several nonrandomised studies suggested that etiologic treatment led to negative serological tests and prevented clinical and electrocardiographic changes associated with disease progression (Macedo \& Silveira 1987, Miranda et al. 1994, Viotti et al. 1994, 2006, Fragata Filho et al. 1995, Fabbro et al. 2000, Gallerano \& Sosa 2001).

However, other studies yielded contradictory results, concluding that the etiologic treatment in the chronic phase of Chagas disease failed to exterminate the parasite, halt the progress of the disease, or prevent its complications (Ianni et al. 1993, Catalioti \& Acquatella 1999, Lauria-Pires et al. 2000, Britto et al. 2001).

Regardless of the results, the evidence from these nonrandomised studies is weak, as shown in the best of these investigations the 2006 paper by Viotti et al. In this trial, 598 of the 1,968 screened chronic chagasic patients were unblindly and non-randomly assigned to no treatment or to treatment with $5 \mathrm{mg} / \mathrm{kg}$ per day of benznidazole for 30 days. Nearly 60 patients in each group were lost to follow-up. In those remaining in the trial, after a median follow-up period of approximately 11.5 years, conversion to negative results on serologic tests was more frequent in treated patients than in untreated patients [32 of $218(15 \%)$ vs. 12 of $212(6 \%)$; adjusted hazard ratio, 2.1 (CI, 1.06-4.06); $\mathrm{p}=0.034]$. Also, fewer treated patients showed progression of disease [12 of 283 (4\%) vs. 40 of 283 (14\%); adjusted hazard ratio, 0.24 (95\% CI, 0.10-0.59); $\mathrm{p}=0.002]$ or developed abnormalities on electrocardiography [15 of $283(5 \%)$ vs. 45 of $283(16 \%)$; adjusted hazard ratio, 0.27 (CI, 0.13-0.57); $\mathrm{p}=0.001$ ) compared to untreated patients (Viotti et al. 2006).

Before the publication of that last trial, two independent analyses of pooled data from several of these observational studies concluded that there was insufficient evidence to support the routine use of trypanocidal therapy in patients with chronic Chagas disease (Villar 2002, Reyes \& Vallejo 2005). 


\section{Evidence from randomised clinical trials}

After a systematic review of the literature, we found only five randomised trials investigating the effects of trypanocidal therapy in chronic Chagasic patients (Villar et al. 2002). In all, there were 756 patients whose data were pooled and used for a meta analysis. Unfortunately, only three of these studies used benznidazole or nifurtimox as trypanocidal agents (Andrade et al. 1996, Coura et al. 1997, Sosa Estani et al. 1998), while the other studies used ineffective drugs, such as allopurinol, skewing their results (Lauria-Pires et al. 1988, Rassi et al. 2007). Furthermore, none of these studies used definitive hard clinical endpoints. Nevertheless, after a follow-up period ranging from 1-4 years, several indices showed significantly less parasite burden in treated patients compared to untreated patients. Negative seroconversion occurred in 61 of the 102 treated patients, compared to only six of the 98 untreated patients [OR (odds ratio) $=10.91, \mathrm{p}<0.01)$. Forty of the 42 treated patients (95\%) also had a negative xenodiagnosis compared to only 21 of the 43 untreated patients $(48 \%$; OR $=5.37$, $\mathrm{p}<0.01$ ). Additionally, when looking at the reduction of antibody titres against the T. cruzi, the OR comparing the treated vs. the non-treated groups was $0.54(\mathrm{p}<$ $0.01)$. Finally, ECG changes - the only clinical endpoint reported - were seen in fewer treated patients (2/99) than non-treated patients (5/99) (Villar et al. 2002)

These results are encouraging, as they suggest that patients with chronic Chagas disease benefit from trypanocidal treatment, at least in terms of parasite burden reduction. However, the failure of trypanocidal drugs in treating patients in the chronic phase of Chagas disease could be better diagnosed if more sensitive methods for detection, such as PCR, were used (Britto et al. 1995, Galvão et al. 2003).

Overall, the decision to prescribe anti-parasite treatment to patients with chronic Chagas disease is left to the individual physician, as there is no definitive evidence arguing for or against such therapy. This decision is especially important when the patient already exhibits symptoms or signs of cardiomyopathy. The physician opting not to offer trypanocidal therapy is running the risk of committing the type II or $\beta$-error. Conversely, adopting the etiologic therapy alternative, the physician runs the risk of type I or $\alpha$-error. The only way to resolve this dilemma is to directly examine the effect of antiparasitic treatment on the clinical outcome of chronic Chagas disease. Therefore, the BENEFIT trial - Benznidazole Evaluation for Interrupting Trypanosomiasis - was undertaken to test the hypothesis that eliminating the etiologic agent or at least reducing the parasite burden, may favourably impact the clinical evolution of patients with Chagas-induced cardiomyopathy. The design and rationale of this study has been recently published (Marin-Neto et al. 2008).

\section{The BENEFIT project}

Except for the initial period of drug trial administration, during which the patients will be double blindly treated with the active drug or placebo, the protocol is designed to not interference with the management of the clinical manifestations of Chagas-induced cardiomyopathy, including arrhythmia, heart failure or thromboembolism. The two parallel treatment groups are composed of both men and women aged 18-75 years. The women were not pregnant and had no childbearing potential. Broad inclusion indications, with few exclusion criteria, were selected to assure a fully representative sample of the many types of patients with chronic Chagas cardiomyopathy. The trial will include two consecutive phases.

\section{The BENEFIT pilot study}

The primary objective of the BENEFIT pilot study will be to determine the efficacy of benznidazole in reducing parasite burden in patients with chronic Chagas cardiomyopathy, as well as assess the drug's safety and tolerability.

The co-primary efficacy outcomes of the pilot study are negative $T$. cruzi detection by PCR and mean parasite load reduction as assessed by the concentration of $T$. cruzi $/ \mathrm{mL}$ of blood by real-time PCR.

There are three secondary outcomes for the BENEFIT pilot study: (i) safety and tolerability of benznidazole, as determined by the incidence of adverse effects reported in each group and the number who complete the treatment period at full dose. The target for the latter outcome is $85 \%$ completion of therapy in the treated group at the end of the treatment period. Safety and tolerability assessment will determine the incidence of minor symptoms such as malaise, headache, gastric or enteric intolerance, and more significant adverse effects, such as dermatitis, lowering of white blood cell counts and peripheral neuropathy. Toxic effects will also be assessed through liver and renal function tests [aspartate amino-transferase (AST), alanine amino-transferase (ALT), creatinine] measured before initiation and after completion of treatment. The number of leucocytes will be assessed after three weeks of treatment and after completion of therapy. The incidence of drug discontinuation will be recorded in the two experimental groups [brief $(<1$ week) interruption is allowed for minor intolerance]. The only strict criteria for permanent discontinuation of benznidazole study medication are: significant leucopoenia $(<2,500)$, peripheral neuropathy or severe allergic dermopathy (not responding to corticosteroids). In other situations, discontinuation of the study drug will be discouraged as much as possible. Study drug accountability, as well as all pre-specified concomitant medications, will be appropriately recorded at each scheduled visit; (ii) long-term feasibility, which will be assessed by patient enrolment and patient completion of follow-up. The target for completeness of follow-up at two years is $95 \%$ and (iii) composite of clinically significant outcomes, which include death, cardiac transplantation, resuscitated cardiac arrest, documented sustained ventricular tachycardia (SVT) requiring cardioversion, new development of symptomatic congestive heart failure, implantation of pacemaker or cardiac defibrillator, stroke or any other systemic of pulmonary thromboembolic event in patients with no prior thromboembolism. 


\section{The BENEFIT full-scale trial}

The primary objective of the full-scale trial is to evaluate whether antitrypanosomal therapy with benznidazole reduces mortality and major cardiovascular clinical outcomes in patients with chronic Chagas heart disease. The primary outcome of the BENEFIT full-scale is a composite of major cardiovascular outcomes defined as the first occurrence of death, resuscitated cardiac arrest, SVT, symptomatic heart failure, pacemaker or cardiac defibrillator insertion, stroke or other systemic or pulmonary thromboembolic event. The secondary objective of the BENEFIT full-scale trial is to determine if the etiologic treatment reverses or halts objective evidence of left ventricular (LV) function deterioration and ECGchanges, or reduces symptoms and parasite burden. The secondary outcomes for the BENEFIT full-scale trial are: (i) new development of any of the following echo changes: segmental wall motion abnormalities (including ventricular aneurysm), reduction in LV ejection fraction $>$ $5 \%$, increase in LV diastolic diameter (LVDD) $>5.0 \mathrm{~mm}$ compared with baseline; (ii) new 12-lead ECG alterations; (iii) progression of New York Heart Association (NYHA) functional class by at least one category and (iv) reduction in parasite burden as assessed by real-time PCR.

A number of substudies will be conducted as part of the BENEFIT program and will address other issues of parasite pathogenicity, their relationship with geographical location and describe the clinical course in populations that are infected by different strains of the parasite such as T. cruzi I and II (Prata 2001).

\section{Patient eligibility criteria}

Patients with confirmed Chagas disease, i.e., any combination of at least two positive serological tests for Chagas disease (indirect immunofluorescence, indirect hemagglutination or ELISA), aged $\geq 18$ years and $\leq 75$ years are eligible to participate in the BENEFIT trials if they have evidence of cardiomyopathy based on one or more of the following criteria: (i) abnormal ECG (at least 2 of the following changes): right bundle branch block; left bundle branch block; left anterior fascicular block; left posterior fascicular block; ventricular premature beats; first degree atrioventricular (AV) block $>220 \mathrm{~m}$ in the absence of drugs that slow AV conduction; Mobitz type I AV block in the absence of drugs that slow AV conduction; sinus bradycardia $<50 \mathrm{bpm}$ or sinus pauses $>3 \mathrm{~s}$ in the absence of sinus node-blocking drugs; primary ST-T changes; abnormal Q waves; low voltage of QRS or (ii) atrial fibrillation or abnormal ECG (only 1 of the following changes is necessary: Mobitz type II advanced or (iii) third degree AV block and cardiac pacemaker or (iii) implanted automatic defibrillator or (iv) increased cardiothoracic ratio $(>0.50)$ or $(v)$ complex ventricular arrhythmias (multiform $>10 \mathrm{~h}$, couplets or non-SVT on $24 \mathrm{~h}$ ambulatory ECG monitoring or (vi) evidence of regional wall-motion abnormality or reduced $(<50 \%)$ global LV systolic function (2D-Echo, radionuclide angiography, contrast ventriculography) or increased LV end diastolic diameter ( $>55 \mathrm{~mm}$ ) on 2D-Echo.
A few exclusion criteria are applicable to enrolling patients: NYHA heart failure class IV or decompensated heart failure; evidence of concomitant coronary artery disease or other aetiology of dilated cardiomyopathy; previous treatment with trypanocidal agents or an accepted indication for antiparasitic therapy (e.g. reactivation of Chagas infection due to immunosuppression by several diseases or treatment with steroids); inability to comply with follow-up; history of severe alcohol abuse within two years; known chronic renal insufficiency (serum creatinine $>2.5 \mathrm{mg} / \mathrm{dL}$ or $200 \mu \mathrm{moL}$ ) or hepatic insufficiency (AST/ALT $>3 \times$ normal); pregnancy or breast feeding; megaesophagus with severe swallowing impairment; or other severe disease significantly curtailing life expectancy.

\section{Ethics and patient confidentiality}

All the procedures used are in accordance with the ethical standards of the responsible committees on human experimentation (institutional for each centre and regional for each country involved) and with the Helsinki Declaration of 1975, as revised in 1983).

\section{Sample sizes}

Six hundred patients with positive parasite detection by PCR at baseline will be recruited in the pilot trial. A spontaneous negativisation rate of $20-30 \%$ is expected in the patients receiving placebo (Britto et al. 1995, 2001, Galvão et al. 2003), The sample size calculations were done with two possible treatment effects, a 50\% and a $100 \%$ relative increase in negativisation for the two expected rates of spontaneous negativisation $(20 \%$ and $30 \%$ ). With a 2 -sided $\alpha=0.04$ in this analysis, there is excellent power to detect a doubling of negativisation (increase of $100 \%$ ) and reasonable power to detect a $50 \%$ increase in negativisation within the range of spontaneous negativisation expected in the control group. The pilot study is well-powered to detect a reduction in mean parasite load between the two groups after two years, and a relative reduction of $25 \%$ in parasite load (or an absolute reduction of 6.5) with benznidazole.

Three thousand patients are needed for the fullscale trial (1,500 per group). This represents the minimum number required to detect a $26 \%$ relative risk in the risk of the composite end point (death, resuscitated cardiac arrest, cardiac transplantation, development of new heart failure, life-threatening nonfatal arrhythmias, thromboembolism and need for pacemaker or defibrillator implantation) with $90 \%$ power. This calculation assumes a yearly event rate of $8 \%$ in the control group and 4-6 years of follow-up (at 2-sided $\alpha=0.05$ ) (Rassi et al. 2006). The reported rates of noncompliance with benznidazole are around $17 \%$ and we expect a loss to follow-up of $3 \%$.

\section{Treatment regimen and follow-up}

The first 1,500 patients were randomly assigned to placebo or benznidazole at $5 \mathrm{mg} / \mathrm{kg}$ per day for 60 days, with an upper limit of dosing at $400 \mathrm{mg}$ per day (corresponding to a body weight of $80 \mathrm{~kg}$ ). For the last 1,500 
patients, due to logistic reasons, the dosing policy was changed. While keeping the rate of $5 \mathrm{mg} / \mathrm{kg}$ of body weight and the total dose to be taken, we will limit the maximum daily dose of $300 \mathrm{mg}$ per day and establish a direct relationship between the number of days of treatment and the body weight. Thus, a $60 \mathrm{~kg}$ patient will take $300 \mathrm{mg}$ for 60 days, a $80 \mathrm{~kg}$ patient with will take 300 mg per day for 80 days and a $40 \mathrm{~kg}$ patient will take 300 mg per day for 40 days.

Randomisation is always at 1:1, with stratification according to centre, using a random-block system. Scheduled follow-up visits will occur at 11 days after initiation of therapy, 21 days, and at the completion of treatment. The next visits will be at six months and then annually until a minimum of four and a maximum of six years have passed.

\section{Data analysis}

All analyses of primary and secondary outcomes will be performed according to the intention-to-treat principle. The pilot study will also determine the actual event rate in this population. For the pilot study, the rates of negativisation of parasite detection by PCR (first co-primary outcome) in the benznidazole will be compared to the placebo groups. Logistic regression will be performed for the rate of negativisation at two years of follow-up. The second co-primary outcome, the difference in parasite load between the two treatment groups at two years of follow-up, will be tested using analysis of variance techniques at a 2 -sided $\alpha$ of 0.01 .

For the full-scale study, the primary analyses will compare the time to the first occurrence of any element of the primary composite outcome. Patients lost to follow-up will be censored at the last time of observation. Cox proportional hazards model will be used to investigate the influence of important confounders and prognostic factors. A sensitivity analysis will be performed in patients with prior SVT, previous insertion of pacemaker or defibrillator, thromboembolic phenomena or heart failure hospitalisation. A prespecified subgroup analysis based on the severity of chronic chagasic cardiomyopathy (CCC) at admission will also be performed. Severity of CCC will be graded according to the recently developed Rassi score (Rassi et al. 2006).

\section{Trial progress}

Recruitment was initiated in November of 2004 and currently includes 42 centres in Argentina, Bolivia, Brazil and Colombia. As of 18 June 2009, over 1,600 patients have been enrolled. The overall cumulative rate of drug interruptions is nearly $15 \%$, with approximately $6 \%$ of these patients restarting the assigned treatment.

In conclusion, the BENEFIT trial addresses the unanswered question of whether etiologic treatment is beneficial for patients already presenting with clinical manifestations of Chagas heart disease. The randomised, controlled format of this study constitutes the best tool to provide evidence to support any therapeutic decision.

Some might wonder why this trial did not focus on the more prevalent population of chagasic patients, i.e., those with the indeterminate form of chronic Chagas disease. The very long-lasting and unpredictable course of this form of the disease makes any study in this patient population difficult. A more prolonged follow-up period in a larger sample size would be required to get a sufficient number of hard clinical events to reasonably test any hypothesis. However, if the BENEFIT trial succeeds in showing that trypanocidal therapy effectively improves the clinical course of patients with overt cardiomyopathy, we could sensibly extrapolate that etiologic treatment might also protect patients with the indeterminate form of chronic Chagas disease.

\section{REFERENCES}

Amorim DS, Marin-Neto JA 1995. Functional alterations of the autonomic nervous system in Chagas' heart disease. São Paulo Med $J$ 113: 772-784.

Andrade AL, Zicker F, de Oliveira RM, Almeida Silva S, Luquetti A, Travassos LR, Almeida IC, De Andrade SS, de Andrade JG, Martelli CM 1996. Randomized trial of efficacy of benznidazole in treatment of early Trypanosoma cruzi infection. Lancet 348 : 1407-1413.

Andrade SG, Stocker-Guerret S, Pimentel AS, Grimaud JA 1991. Reversibility of cardiac fibrosis in mice chronically infected with Trypanosoma cruzi, under specific chemotherapy. Mem Inst Oswaldo Cruz 86: 187-200.

Bellotti G, Bocchi E, de Moraes AV, Higuchi ML, Barbero-Marcial M, Sosa E, Esteves-Filho A, Kalil R, Weiss R, Jatene A, Pileggi F 1996. In vivo detection of $T$. cruzi antigens in hearts of patients with chronic Chagas' heart disease. Am Heart J 131: 301-307.

Bern C, Montgomery SP, Herwaldt BL, Rassi A Jr, Marin-Neto JA, Dantas RO, Maguire JH, Acquatella H, Morillo C, Kirchhoff LV, Gilman RH, Reyes PA, Salvatella R, Moore AC 2007. Evaluation and treatment of Chagas disease in the United States. A systematic review. JAMA 298: 2171-2181.

Britto C, Cardoso MA, Vanni CM, Hasslocher-Moreno A, Xavier SS, Oelemann W, Santoro A, Pirmez C, Morel CM, Wincker P 1995. Polymerase chain reaction detection of Trypanosoma cruzi in human blood samples as a tool for diagnosis and treatment evaluation. Parasitology 110: 241-247.

Britto C, Silveira C, Cardoso MA, Marques P, Luquetti A, Macedo V, Fernandes O 2001. Parasite persistence in treated chagasic patients revealed by xenodiagnosis and polymerase chain reaction. Mem Inst Oswaldo Cruz 96: 823-826.

Catalioti F, Acquatella H 1999. Comparación de mortalidad durante seguimiento por 5 años en sujetos con enfermedad de Chagas crónica con y sin tratamiento de benznidazol. Primer simposio virtual en enfermedad de Chagas. Available from: http://pcvc. sminter.com.ar/cvirtual/tlibres/tnn2574/tnn2574.htm.

Chagas C 1909. Über eine neue trypanosomiasis des Menschen. Archiv Schiffs Tropen Hyg 13: 5-7.

Coura JR 2007. Chagas disease: what is known and what is needed. A background article. Mem Inst Oswaldo Cruz 102 (Suppl. I): 113-122.

Coura JR, de Abreu LL, Willcox HP, Petana W 1997. Comparative controlled study on the use of benznidazole, nifurtimox and placebo in the chronic form of Chagas disease, in a field area with interrupted transmission. I. Preliminary evaluation. Rev Soc Bras Med Trop 30: 139-144.

Cunha-Neto E, Bilate AM, Hyland KV, Fonseca SG, Kalil J, Engman DM 2006. Induction of cardiac autoimmunity in Chagas heart disease: a case for molecular mimicry. Autoimmunity 39: 41-54. 
Dias JC, Prata A, Correia D 2008. Problems and perspectives for Chagas disease control: in search of a realistic analysis. Rev Soc Bras Med Trop 41: 193-196.

Dias JCP 1989. The indeterminate form of human chronic Chagas' disease: a clinical epidemiological review. Rev Soc Bras Med Trop 22: 147-156.

Fabbro De Suasnábar D, Arias E, Streiger M, Piacenza M, Ingaramo M, Del Barco M, Amicone N 2000. Evolutive behavior towards cardiomyopathy of treated (nifurtimox or benznidazole) and untreated chronic chagasic patients. Rev Inst Med Trop Sao Paulo 42: 99-109.

Fragata Filho AA, Boianain E, Silva MAD, Correia EB, Borges Filho R, Martins C, Salene V, Batlouni M, Souza E 1995. Validade do tratamento etiológico da fase crônica da doença de Chagas com benznidazol. Arq Bras Cardiol 65 (Suppl. I): 71.

Gallerano RH, Sosa RR 2001. Resultados de estúdio a largo plazo com drogas antiparasitárias em infectados chagásicos crônicos. Rev Fed Arg Cardiol 30: 289-296.

Galvao LM, Chiari E, Macedo AM, Luquetti AO, Silva SA, Andrade AL 2003. PCR assay for monitoring Trypanosoma cruzi parasitemia in childhood after specific chemotherapy. J Clin Microbiol 41: 5066-5070.

Garcia S, Ramos CO, Senra JFV, Vilas-Boas F, Rodrigues MM, Campos-de-Carvalho AC, Ribeiro-Dos-Santos R, Soares MB 2005. Treatment with benznidazole during the chronic phase of experimental $\mathrm{CD}$ decreases cardiac alterations. Antimicrob Agents Chemother 49: 1521-1528.

Ianni BM, Arteaga E, Mady C, Barretto ACP, Pileggi F 1993. Uso de benznidazol em chagásicos na forma indeterminada: resultados a longo prazo. Arq Bras Cardiol 61 (Suppl. II): 130.

Kierszenbaum F 2007. Mechanisms of pathogenesis in Chagas disease. Acta Parasitol 52: 1-12.

Köberle F 1968. Chagas' heart disease and Chagas' syndromes: the pathology of American trypanosomiasis. Adv Parasitol 6: 63-116.

Lauria-Pires L, Braga MS, Vexenat AC, Nitz N, Simões-Barbosa A, Tinoco DL, Teixeira AR 2000. Progressive chronic Chagas heart disease ten years after treatment with anti-Trypanosoma cruzi nitroderivatives. Am J Trop Med Hyg 63: 111-118.

Lauria-Pires L, de Castro CN, Emanuel A, Prata A 1988. Ineffectiveness of allopurinol in patients in the acute phase of Chagas disease. Rev Soc Bras Med Trop 21: 79.

Macedo VO, Silveira CA 1987. Perspectivas da terapêutica específica na doença de Chagas. Experiências na forma indeterminada. Rev Soc Bras Med Trop 20 (Suppl. II): M24-M26.

Marin-Neto JA, Cunha-Neto E, Maciel BC, Simoes MV 2007. Pathogenesis of chronic Chagas heart disease. Circulation 115: 1109-1123.

Marin-Neto JA, Marzullo P, Marcassa C, Gallo Jr L, Maciel BC, Bellina CR, L'Abbate AL 1992. Myocardial perfusion abnormali- ties in chronic Chagas' disease as detected by thallium-201 scintigraphy. Am J Cardiol 69: 780-784.

Marin-Neto JA, Rassi A Jr, Morillo CA, Avezum A, Connolly SJ, Sosa-Estani S, Rosas F, Yusuf S 2008. BENEFIT investigators. Rationale and design of a randomized placebo-controlled trial assessing the effects of etiologic treatment in Chagas' cardiomyopathy: the BENznidazole Evaluation For Interrupting Trypanosomiasis (BENEFIT). Am Heart J 156: 37-43.

Miranda L, Miranda L, Campos G, Marins N, Lobo V 1994. História natural da forma crônica da doença de Chagas $\mathrm{x}$ tratamento específico. RECOC 1: 25-29.

Prata A 2001. Clinical and epidemiological aspects of Chagas disease. Lancet Infect Dis 1: 92-100.

Rassi A, Luquetti AO, Rassi A Jr, Rassi GG, Rassi SG, DA Silva IG, Rassi AG 2007. Specific treatment for Trypanosoma cruzi: lack of efficacy of allopurinol in the human chronic phase of Chagas disease. Am J Trop Med Hyg 76: 58-61.

Rassi A, Rassi Jr. A, Rassi GG 2000. Fase aguda. In: Z Brener, ZA Andrade, M Barral-Neto (eds.), Trypanosoma cruzi e doença de Chagas, Guanabara Koogan, Rio de Janeiro, p. 231-245.

Rassi Jr A, Rassi A, Little WC 2006. Development and validation of a risk score for predicting death in Chagas' heart disease. $N E n g l$ J Med 355: 799-808.

Reyes PA, Vallejo M 2005. Trypanocidal drugs for late stage, symptomatic CD (Trypanosoma cruzi infection). Cochrane Database Syst Rev: CD004102.

Rossi MA 1990. Microvascular changes as a cause of chronic cardiomyopathy in Chagas' disease. Am Heart J 120: 233-236.

Sosa Estani S, Segura EL, Ruiz AM, Velazquez E, Porcel BM, Yampotis C 1998. Efficacy of chemotherapy with benznidazole in children in the indeterminate phase of Chagas disease. Am J Trop Med Hyg 59: 526-529.

Tarleton RL 2003. Trypanosoma cruzi and Chagas disease: cause and effect. In: KM Tyler, MA Miles (eds.), World class parasites: American trypanosomiasis, Vol. 7, Kluwer Academic Publisher, Norwell, p. 107-116.

Villar JC 2002. Desenlaces clínicos de sujetos com infección crônica por Trypanosoma cruzi tratados o no con agentes tripanocidas. Un metaanálisis de estúdios observacionales. MEDUNAB 5: 166-173.

Villar JC, Marin-Neto JA, Ebrahim S, Yusuf S 2002. Trypanocidal drugs for chronic asymptomatic Trypanosoma cruzi infection. Cochrane Database Syst Rev: CD003463.

Viotti R, Vigliano C, Armenti H, Segura E 1994. Treatment of chronic Chagas disease with benznidazole: clinical and serologic evolution of patients with long-term follow-up. Am Heart J 127: 151-162.

Viotti R, Vigliano C, Lococo B, Bertocchi G, Petti M, Alvarez MG, Postan M, Armenti A 2006. Long-term cardiac outcomes of treating chronic $\mathrm{CD}$ with benznidazole versus no treatment: a nonrandomized trial. Ann Intern Med 144: 724-734. 